\title{
On the detection of the first extragalactic classical chemically peculiar stars ${ }^{\star}$
}

\author{
H. M. Maitzen ${ }^{1}$, E. Paunzen ${ }^{1,2}$, and O. I. Pintado ${ }^{3, \star \star}$ \\ 1 Institut für Astronomie der Universität Wien, Türkenschanzstr. 17, 1180 Wien, Austria \\ 2 Zentraler Informatikdienst der Universität Wien, Universitätsstr. 7, 1010 Wien, Austria \\ 3 Departamento de Física, Facultad de Ciencias Exactas y Tecnología, Universidad Nacional de Tucumán, \\ Argentina - Consejo Nacional de Investigaciones Científicas y Técnicas de la República Argentina
}

Received 16 January 2001 / Accepted 22 March 2001

\begin{abstract}
We report on the detection of the first extragalactic, classical chemically peculiar (CP) stars more than 100 years after the discovery of this group. Using the tool of CCD $\Delta a$-photometry, eight definite positive detections in the globular-like stellar cluster NGC 1866 and its surrounding in the Large Magellanic Cloud were made. More than 1950 objects within a radius of $9.5^{\prime}$ of the cluster center down to 20.5 magnitude were observed in this three filter, narrow band photometric system. Averaging more than 70 individual frames for all three filters yielded a $3 \sigma$ detection limit of $0.015 \mathrm{mag}$ for stars with $V=20 \mathrm{mag}$. Furthermore we found three objects with significantly negative $\Delta a$-values indicating either an $\mathrm{Ae} /$ shell or $\lambda$ Bootis nature. The overall percentage of chemically peculiar stars for NGC 1866 (1.5\%) seems lower than for the majority of open clusters in our Milky Way whereas the incidence within the LMC field is decisively less $(0.3 \%)$.
\end{abstract}

Key words. galaxies: magellanic clouds - stars: chemically peculiar - stars: early-type

\section{Introduction}

Observational evidence has shown that on the average $5 \%$ of the main sequence objects in the range B2 to F2 are magnetic peculiar stars with a maximum frequency among the late B-type stars of about $10 \%$. Subsequently, these stars revealed other peculiar features, e.g. the existence of a strong global magnetic field with a predominant dipole component located at random with respect to the stellar rotation axis and the center of the star.

The origin of the magnetic fields is still a matter of debate: those who favor the survival of frozen-in fossil fields originating from the medium out of which the stars were

Send offprint requests to: E. Paunzen, e-mail: Ernst.Paunzen@univie.ac.at

* Based on observations obtained at Complejo Astronómico el Leoncito (CASLEO), operated under the agreement between the Consejo Nacional de Investigaciones Científicas y Técnicas de la República Argentina and the National Universities of La Plata, Córdoba y San Juan.

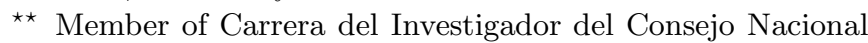
de Investigaciones Científicas y Técnicas de la República Argentina. formed are in opposition to those following the idea that a dynamo mechanism is acting in the interior of these stars.

A plausible diagnostic tool for the investigation of the formation of magnetic peculiar stars from an observational point of view is their statistical identification in a variety of galactic as well as extragalactic environments, implying both different metallicities and different interstellar magnetic field strengths. Star formation in the Large Magellanic Cloud (LMC hereafter) has been proceeding in a different way as compared to our Galaxy. The lower metallicity has seemingly favoured the formation of young populous (globular-like) stellar clusters general not present in our own Galaxy.

As a tool for the detection of distant magnetic peculiar stars we have used CCD filter photometry in the narrow band $\Delta a$-system introduced by Maitzen (1976). This system samples the broad band flux depression around $520 \mathrm{~nm}$, characteristic of magnetic peculiar stars (in the notation of Preston 1974 they form the CP2 group with an admixture of magnetic helium weak stars - CP4). This is done through measurements in three filters: $g_{1}$ at $500 \mathrm{~nm}$ and $y$ at $550 \mathrm{~nm}$, both representing the continuum at the edges of the feature, and $g_{2}$ located at the center of the 

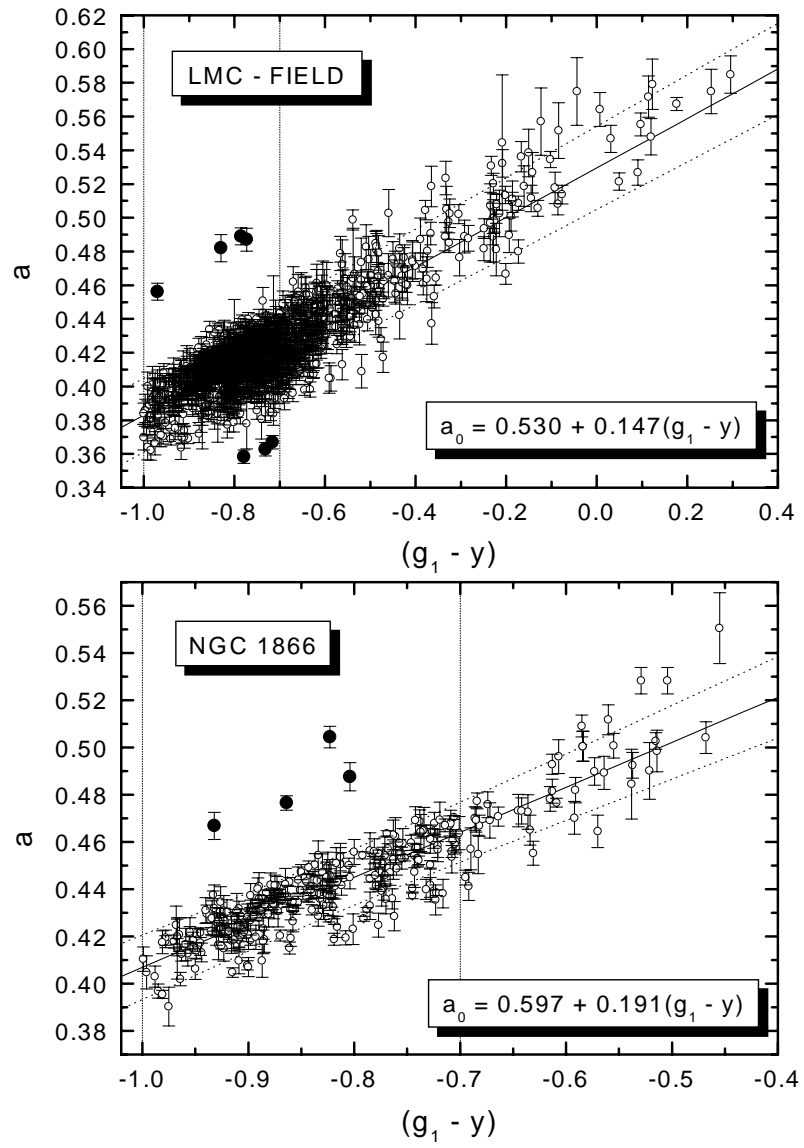

Fig. 1. $a$ versus $\left(g_{1}-y\right)$ for NGC 1866 (lower panel; field 1) and its surrounding (upper panel; field 2). Filled circles indicate apparent peculiar stars whereas open circles are non-peculiar objects. The vertical lines denote the spectral type range (B8 to F2) where chemical peculiar stars are expected. The full line is the normality line whereas the dotted lines are the confidence intervals corresponding to 99.9 percent

feature at $521 \mathrm{~nm} . \Delta a$ is the magnitude difference of $g_{2}$ relative to the mean of $g_{1}$ and $y$, normalized to zero for a non-peculiar star $\left(a=g_{2}-\left[\left(g_{1}+y\right) / 2\right]\right)$. The photometric accuracy needed to detect classical peculiar stars is typically a few mmags. It has been often demonstrated (e.g. Vogt et al. 1998) that this technique is equivalent to spectroscopic detection at classification dispersion. A detailed description of the $\Delta a$ photometric system can be found in Bayer at al. (2000).

The efficiency of CCD-photometry in the $\Delta a$-system for detecting CP2 stars both in the field and in the open clusters has been demonstrated recently by Maitzen et al. (1997) and Bayer et al. (2000), even using a small, $60 \mathrm{~cm}$ telescope.

At CASLEO we have obtained $\Delta a$-photometry of NGC $1866(\log t=8.0,[\mathrm{Fe} / \mathrm{H}]=-0.4 \mathrm{dex})$, a "key cluster" for abundance determinations, stellar evolution theory and study of $\delta$ Cephei stars, and its surrounding. We obtained $\Delta a$-photometry for 1979 stars brighter than $20.5 \mathrm{mag}$. The possible finding of peculiar stars as well as the color-magnitude diagram are discussed in the following.

\section{Observation, reduction and calibration}

The observations were performed at the Complejo Astronómico el Leoncito (CASLEO) using the $2.15 \mathrm{~m}$ telescope (observer: O. I. Pintado). With a TEK-1024 CCD and a focal reducer, a scaling of $0.813^{\prime \prime}$ pixel $^{-1}$ was achieved. Since our filters do not cover the whole CCDchip, an effective field of view of about $9.5^{\prime}$ was observed.

During four consecutive nights from November 20th to 23rd, 1998, NGC 1866 and its surrounding were photometrically investigated. Furthermore, NGC 3114, a well studied open cluster in our Milky Way was used as a further test of our photometric system. The typical integration time was about five to ten minutes. A detailed observing $\log$ can be requested from the second author.

In total 76 frames in all filters for the field of NGC 1866 and its surrounding were obtained. The characteristics of the filters have been described in Bayer et al. (2000).

The basic reductions (bias-subtraction, darkcorrection, flat-fielding) were carried out within standard IRAF routines. A point-spread-function-fitting procedure within the IRAF task DAOPHOT was performed, typically more than 30 individual, single stars were used to derive the PSF for each frame. Because of instrumentally induced offsets and different airmasses between the single frames, photometric reduction of each frame was performed separately and the measurements were then averaged and weighted by their individual photometric error. This is justified because the "standard" as well as program stars are on the same frame.

The table of all program stars with their photometric quantities and corresponding errors are only available at SIMBAD or from the second author upon request.

Unfortunately it was not possible to use the Strömgren uvby $\beta$ photometry from Hilker et al. (1995) for the calibration of our photometric values because it neither has been available in electronic form nor upon request from the authors of the given reference. Furthermore, no printed tables are available.

In order to check our results and to transform $y$, we have used the Johnson $U B V$ colors from Testa et al. (1999). They observed an almost identical, but smaller field $\left(6^{\prime} \times 6^{\prime}\right)$ centered on NGC 1866 reaching a magnitude limit of about $23 \mathrm{mag}$ with a scaling of $0.363^{\prime \prime}$ pixel $^{-1}$.

For the calibration of $y$ to standard Johnson $V$ values, we chose stars without any other objects within a radius of $3^{\prime \prime}$ resulting in 959 stars common with the list of Testa et al. (1999). A linear least square fit resulted in:

$V=-5.130(17)+1.027(8) \cdot y$.

In parenthesis are the errors in the final digits of the corresponding quantity. The same stars were used to estimate the $\left(g_{1}-y\right)$ range for which classical chemical peculiar stars are expected (spectral range B8 to F2) resulting in $-1.0<\left(g_{1}-y\right)<-0.7 \mathrm{mag}$.

For the discussion of the percentage of peculiar stars compared to normal ones, the field was divided in the innermost regions of NGC 1866 (1.5', including annulus 1 to 

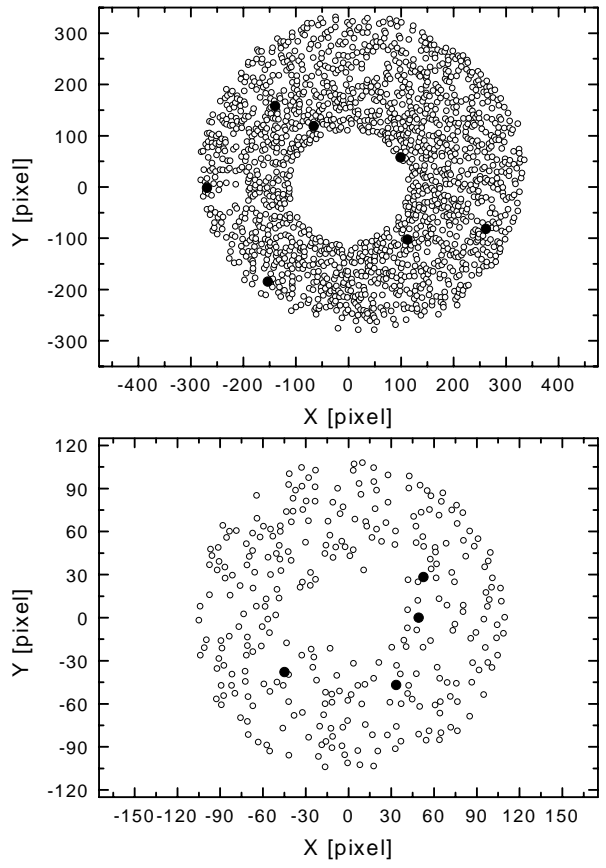

Fig. 2. Position of normal (open circles) and apparent peculiar stars (filled circles) for NGC 1866 (lower panel) and its surrounding (upper panel). North is upwards, east is to the right; 1 pixel $=0.813^{\prime \prime} ; X=Y=0$ corresponds to $\alpha(2000)=05^{\mathrm{h}} 13^{\mathrm{m}} 40^{\mathrm{s}}$ and $\delta(2000)=-65^{\circ} 27^{\prime} 49^{\prime \prime}$

3 from Testa et al. 1999; field 1 hereafter) and its surrounding (up to $9.5^{\prime}$; field 2 hereafter). Due to severe crowding, no objects could be resolved within a radius of $20^{\prime \prime}$ of the center.

For the further analysis, stars were only considered with no other object within a radius of 6 pixels (or $4.8^{\prime \prime}$ ) and/or a nearby object which is fainter than two magnitudes using the lists of Testa et al. (1999). The latter guarantees that no severe contamination occurs resulting in 301 as well as 1678 objects in the corresponding fields.

\section{Results}

Figure 1 shows the $a$ versus $\left(g_{1}-y\right)$ diagrams for both fields. Note that the reddening for NGC 1866 is $E(B-$ $V)=+0.07 \mathrm{mag}$ (Hilker et al. 1995). Excluding the apparent peculiar objects (filled circles in Figs. 1 and 2), the following normality lines for field 1 and 2, respectively, were derived:

$$
\begin{aligned}
& a_{0}=+0.597(8)+0.191(6) \cdot\left(g_{1}-y\right) \\
& a_{0}=+0.530(10)+0.147(5) \cdot\left(g_{1}-y\right) .
\end{aligned}
$$

The scatter of $\Delta a$ around the normality line is very small, resulting in a $3 \sigma$-detection limit of $0.015 \mathrm{mag}$. The different zero-points and slopes are due to the much larger $\left(g_{1}-y\right)$ range of field 2 . Furthermore we note that in field 2, two different populations (cluster and field members of the LMC bulge) are evident (weakly indicated in Fig. 1). These two populations are slightly shifted in $a$ against each other, but their locations do not allow to disentangle individual groups.

We find four objects in each region which are definitely above the normality line with significant positive $\Delta a$ values. Furthermore, three objects in field 2 were found with significant negative $\Delta a$-values. Table 1 lists these stars. From a statistical point of view, the significance ranges from 4.9 to $11.9 \sigma$ (taking the mean photometric error) outside the 99.9 percent confidence interval of the normality line.

Their location in the color-magnitude diagram (Fig. 3) supports membership in LMC. Furthermore, all objects in the common field were unambiguously identified in the list of Testa et al. (1999; note that their observed field is significant smaller than ours). If we assume that these stars are not members of the LMC but of our Milky Way, they have to be white dwarfs because of the necessarily faint absolute magnitude. But for none of them significant spectral features which might produce a positive $\Delta a$-value have been found so far (Koester et al. 1990; Schmidt et al. 1995).

We are, therefore, confident that this means the detection of the first extragalactic CP stars more than 100 years after the discovery of the classical CP stars (for a review see Preston 1974) subject to spectroscopic verification. Another interesting aspect is the detection of three objects with significant negative $\Delta a$-values. These objects might be classical Ae/shell or $\lambda$ Bootis stars (Paunzen $2000)$. Both groups show such a behaviour in the $\Delta a$ photometric system (Maitzen \& Pavlovski 1989a,b; Pavlovski \& Maitzen 1989).

Taking the overall number of observed stars up to $\left(g_{1}-\right.$ $y)=-0.7 \mathrm{mag}$ (spectral type F2; 261 and 1239 objects for field 1 and 2, respectively) and the positive detections, a ratio of about $1.5 \%$ for field 1 can be determined. This is lower than the percentages found in our Milky Way.

The surrounding of NGC 1866 (containing both cluster members and field LMC stars) was treated separately. Here, the overall number of chemically peculiar stars is decisively less $(0.32 \%$ or $0.56 \%$, taking also the objects with negative $\Delta a$-values) than for the cluster center. However, it shows that there are also a few chemically peculiar stars in the field of the LMC.

No systematic trend for the location of these CP stars within field 1 and 2 was found (Fig. 2). This is important because a local interstellar cloud between the Milky Way and the LMC might influence the location of objects within the $a$ versus $\left(g_{1}-y\right)$ diagram.

The results for the field of NGC 3114 proves the high accuracy of our photometric measurements already shown in Bayer at al. (2000). A detailed investigation together with other open clusters of the Milky Way will be published elsewhere.

\section{Conclusion}

High accuracy photometric data in the three filter, narrow band $\Delta a$-system of more than 1950 objects of NGC 1866 
Table 1. Candidate CP stars in the center of NGC 1866 (upper panel; field 1) and in its surrounding (lower panel; field 2). $X=Y=0$ corresponds to $\alpha(2000)=05^{\mathrm{h}} 13^{\mathrm{m}} 40^{\mathrm{s}}$ and $\delta(2000)=-65^{\circ} 27^{\prime} 49^{\prime \prime}$. In parenthesis are the errors in the final digits of the corresponding quantity

\begin{tabular}{rrrrrrrrrrr}
\hline No.1 & \multicolumn{1}{c}{ No.2 } & \multicolumn{1}{c}{$X$} & \multicolumn{1}{c}{$Y$} & $\left(g_{1}-y\right)$ & \multicolumn{1}{c}{$a$} & \multicolumn{1}{c}{$\Delta a$} & \multicolumn{1}{c}{$\sigma_{1}$} & \multicolumn{1}{c}{$V$} & \multicolumn{1}{c}{$N$} \\
\hline 687 & 4986 & -44.75 & -38.01 & $-0.804(8)$ & $0.488(6)$ & +0.044 & 4.9 & $21.459(15)$ & 16.908 & 60 \\
1005 & 9527 & +33.49 & -47.05 & $-0.864(6)$ & $0.477(3)$ & +0.045 & 9.6 & $22.308(4)$ & 17.780 & 71 \\
1073 & 10991 & +49.32 & -0.21 & $-0.823(8)$ & $0.504(5)$ & +0.065 & 10.8 & $24.435(5)$ & 19.965 & 57 \\
1093 & 9448 & +52.65 & +28.10 & $-0.932(8)$ & $0.467(6)$ & +0.048 & 5.7 & $22.763(7)$ & 18.248 & 71 \\
\hline 12 & & -268.74 & -2.22 & $-0.830(10)$ & $0.482(8)$ & +0.074 & 7.4 & $23.698(7)$ & 19.208 & 63 \\
257 & & -153.15 & -184.67 & $-0.716(3)$ & $0.367(4)$ & -0.058 & 10.7 & $21.606(8)$ & 17.059 & 76 \\
304 & 3668 & -139.72 & +157.58 & $-0.732(4)$ & $0.363(4)$ & -0.060 & 11.2 & $24.169(9)$ & 19.692 & 76 \\
595 & 3950 & -65.90 & +118.28 & $-0.779(2)$ & $0.358(4)$ & -0.057 & 10.5 & $22.934(6)$ & 18.423 & 76 \\
1290 & 7563 & +99.51 & +57.63 & $-0.773(6)$ & $0.487(7)$ & +0.071 & 8.2 & $24.285(7)$ & 19.811 & 56 \\
1347 & 5471 & +112.53 & -102.90 & $-0.970(5)$ & $0.456(5)$ & +0.069 & 10.8 & $24.196(10)$ & 19.719 & 64 \\
1862 & & +262.50 & -81.97 & $-0.785(4)$ & $0.489(5)$ & +0.074 & 11.9 & $24.654(9)$ & 20.190 & 45 \\
\hline
\end{tabular}

Cols. 1, 2: Notation for stars sorted after $X$ and $Y$, respectively (Fig. 2) and according to Testa et al. (1999). Cols. 3, 4: $X$ and $Y$-coordinate in pixels (Fig. 2).

Cols. 5, 6: Mean $\left(g_{1}-y\right)$ and $a$-index with the corresponding standard deviation.

Col. 7: Deviation from cluster line $a_{0}=0.597+0.191 \cdot\left(g_{1}-y\right)$ (field 1) or $a_{0}=0.530+0.147 \cdot\left(g_{1}-y\right)$ (field 2).

Col. 8: $\sigma$-value outside the corresponding confidence interval of the normality line.

Cols. 9, 10: Mean $y$ value with its standard deviation and mean $V$ value according to $V=-5.130+1.027 \cdot y$.

Col. 11: Number of measurements for all three filters.

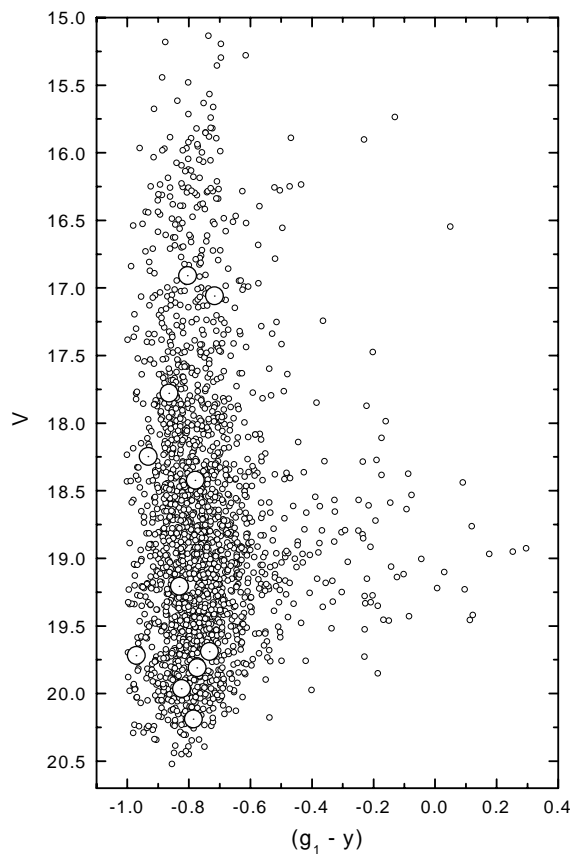

Fig. 3. $V$ versus $\left(g_{1}-y\right)$ for all program stars. The location of apparent peculiar stars (Table 1) has been emphasized

$(\log t=8.0,[\mathrm{Fe} / \mathrm{H}]=-0.4 \mathrm{dex})$ and its surrounding resulted in the photometric detection of the first extragalactic, classical chemical peculiar stars. With a high statistical significance ( 4.9 to $11.9 \sigma$ outside the 99.9 percent confidence interval of the normality line), eight objects with positive and three objects with negative $\Delta a$ values were found. The latter are either Ae/shell or $\lambda$ Bootis candidates. The percentage of peculiar stars for members of NGC 1866 (1.5\%) seems to be lower than the values found for Milky Way clusters (on the average 5\%) whereas the number of the field LMC stars is decisively less $(0.3 \%)$.

As a next step, we suggest to observe these objects spectroscopically with classification dispersion ( $\left.\approx 100 \AA \mathrm{mm}^{-1}\right)$. However, the crowding (especially within the inner parts of NGC 1866) and the relative faintness of the candidates $(16.9<V<20.2 \mathrm{mag})$ challenges the largest telescopes available today.

Acknowledgements. We would like to thank Dr. Testa for providing the photometric data. The authors acknowledge the use of the CCD and data reduction acquisition system supported by US NSF Grant AST 90-15827 to R. M. Rich. OIP acknowledges partial support from grants from the Consejo Nacional de Investigaciones Científicas y Técnicas and Agencia de Promoción Científica of República Argentina. Use was made of the SIMBAD database, operated at CDS, Strasbourg, France.

\section{References}

Bayer, C., Maitzen, H. M., Paunzen, E., Rode-Paunzen, M., \& Sperl, M. 2000, A\&AS 147, 99

Hilker, M., Richtler, T., \& Gieren, W. 1995, A\&A, 294, 648

Koester, D., Wegner, G., \& Kilkenny, D. 1990, ApJ, 350, 329

Maitzen, H. M. 1976, A\&A, 51, 223

Maitzen, H. M., \& Pavlovski, K. 1989a, A\&A, 219, 253

Maitzen, H. M., \& Pavlovski, K. 1989b, A\&AS, 81, 335

Maitzen, H. M., Paunzen, E., \& Rode, M. 1997, A\&A, 327, 636

Paunzen, E. 2000, PASP, 112, 1508

Pavlovski, K., \& Maitzen, H. M. 1989, A\&AS, 77, 351

Preston, G. W. 1974, ARA\&A, 12, 257

Schmidt, G. D., Bergeron, P., \& Fegley, B. 1995, ApJ, 443, 274

Testa, V., Ferraro, F. R., Chieffi, A., et al. 1999, AJ, 118, 2839

Vogt, N., Kerschbaum, F., Maitzen, H. M., \& Faúndez-Abans, M. 1998, A\&AS, 130, 455 